\title{
The SLUGGS Survey: revisiting the correlation between X-ray luminosity and total mass of massive early-type galaxies
}

\author{
Duncan A. Forbes ${ }^{1 \star}$, Adebusola Alabi ${ }^{1}$, Aaron J. Romanowsky ${ }^{2,3}$, Dong-Woo Kim ${ }^{4}$, \\ Jean P. Brodie ${ }^{3}$ and Giuseppina Fabbiano ${ }^{4}$ \\ ${ }^{1}$ Centre for Astrophysics $\mathcal{E}$ Supercomputing, Swinburne University, Hawthorn VIC 3122, Australia \\ ${ }^{2}$ Department of Physics and Astronomy, San José State University, One Washington Square, San Jose, CA 95192, USA \\ ${ }^{3}$ University of California Observatories, 1156 High Street, Santa Cruz, CA 95064, USA \\ ${ }^{4}$ Smithsonian Astrophysical Observatory, 60 Garden Street, Cambridge, MA 02138, USA
}

5 September 2021

\begin{abstract}
Here we utilise recent measures of galaxy total dynamical mass and X-ray gas luminosities $\left(\mathrm{L}_{X, G a s}\right)$ for a sample of 29 massive early-type galaxies from the SLUGGS survey to probe $\mathrm{L}_{X, G a s}$-mass scaling relations. In particular, we investigate scalings with stellar mass, dynamical mass within 5 effective radii $\left(\mathrm{R}_{e}\right)$ and total virial mass. We also compare these relations with predictions from $\Lambda \mathrm{CDM}$ simulations. We find a strong linear relationship between $\mathrm{L}_{X, \text { Gas }}$ and galaxy dynamical mass within $5 \mathrm{R}_{e}$, which is consistent with the recent cosmological simulations of Choi et al. that incorporate mechanical heating from AGN. We conclude that the gas surrounding massive early-type galaxies was shock heated as it fell into collapsing dark matter halos so that $\mathrm{L}_{X, \text { Gas }}$ is primarily driven by the depth of a galaxy's potential well. Heating by an AGN plays an important secondary role in determining $\mathrm{L}_{X, G a s}$.
\end{abstract}

Key words: galaxies: star clusters - galaxies: evolution - galaxies: Xrays

\section{INTRODUCTION}

Massive early-type galaxies (ETGs) reveal halos of hot gas. This $\mathrm{X}$-ray emitting diffuse gas varies in luminosity by a factor of $\sim 100$ 1000 for a given optical luminosity, or equivalently, stellar mass (O’Sullivan, Forbes \& Ponman 2001; Fabbiano 2006; Boroson, Kim \& Fabbiano 2011). It has been suggested that stellar mass loss dominates the source of this gas (Sun et al. 2007; Sarzi et al. 2013). However, in a large X-ray study of ETGs, Goulding et al. (2016) concluded that the data are not consistent with a simple stellar mass loss picture.

An additional source of gas is indicated by the cold dark matter (CDM) paradigm for galaxy formation. Here infalling pristine gas in massive halos is shock-heated to X-ray emitting temperatures as these halos collapse. The hot gas slowly cools while emitting $\mathrm{X}$ rays, with the X-ray luminosity directly related to the depth of the potential well (White \& Frenk 1991). In the GIMIC simulations of Crain et al. (2010), the density of the hot gas is less concentrated than that of the canonical dark matter density profile. This leads to much lower X-ray luminosities than predicted by White \& Frenk (1991), which is then closer to the X-ray luminosity as observed from late-type galaxy halos. However, for early-type galaxies, AGN

^ E-mail: dforbes@swin.edu.au heating of hot gas is also potentially important. Recently, Choi et al. (2015) included AGN in cosmological models of massive ETGs. They found better agreement with the X-ray luminosities of ETGs than when using models without AGN feedback, although some discrepancies remained. These cosmological simulations all suggest that the key parameter determining the luminosity of the diffuse $\mathrm{X}$-ray gas $\left(\mathrm{L}_{X, \text { Gas }}\right)$ is the galaxy mass (which determines the depth of the potential well) with AGN perhaps playing a secondary role in ETGs.

Combining new Chandra data with results from the literature, Kim \& Fabbiano (2013; KF13) have shown that the dynamical (i.e. baryonic plus dark matter) mass may indeed be the key parameter in determining $\mathrm{L}_{X, \text { Gas }}$ surrounding an ETG (see also Mathews et al. 2006). In particular, they showed that measurements of $\mathrm{L}_{X, \text { Gas }}$ correlated strongly with the dynamical mass within $5 \mathrm{R}_{e}$ (where $\mathrm{R}_{e}$ is the effective radius of the galaxy optical light) for a sample of 14 early-type galaxies. The total mass measurements within $5 \mathrm{R}_{e}$ came from the work of Deason et al. (2012) who used a combination of planetary nebulae (PNe) and globular cluster (GC) kinematics from the literature, and the assumption of power-law mass and tracer density profiles.

Here, we use a homogeneous analysis of dynamical masses from GC kinematics within $5 \mathrm{R}_{e}$ for 29 ETGs (Alabi et al. 2017). This analysis is based on data from the SLUGGS survey (Brodie et al. 2014) and supplemented by data from the literature. X-ray 
gas luminosities mostly come from the compilation of Kim \& Fabbiano (2015; hereafter KF15) and are extracted from radii which vary from 30 to $240 \operatorname{arcsec}\left(\sim 2-5 \mathrm{R}_{e}\right.$.) We investigate the trends of the X-ray gas luminosity for our sample with stellar mass, dynamical mass within $5 \mathrm{R}_{e}$ and with extrapolated virial mass. We compare these new observations with simulated galaxy halos from Crain et al. (2010) and Choi et al. (2015), and discuss whether the hot gas halos around ETGs are consistent with an origin, and heating mechanisms, as described by the $\Lambda \mathrm{CDM}$ paradigm.

\section{DATA}

Globular cluster kinematics for galaxies from the SLUGGS survey are supplemented by literature data for NGC 1316, 1399, 4472 and 4636 to give a total sample of 29 galaxies, to derive dynamical masses (Alabi et al. 2017). The SLUGGS (Brodie et al. 2014) survey targets nearby ETGs with stellar masses around $10^{11} \mathrm{M}_{\odot}$. The survey has accumulated over 4000 high precision GC velocities (see e.g. Pota et al. 2013).

Dynamical masses for the sample galaxies have been derived from the GC kinematics by Alabi et al. (2017). They used the tracer mass estimator of Watkins et al. (2010) with the assumption of isotropic orbits for the GCs (mildly radial or tangential orbits give masses within $\sim 10 \%$ of the isotropic case). Small corrections have been made for flattening, rotation or substructures within the GC systems. In Fig. 1 we show a comparison of the total dynamical mass within $5 \mathrm{R}_{e}$ used in this work with that used by KF13, i.e. from Deason et al. (2012). Although there is a reasonable match between the two studies, Fig.1 shows that Alabi et al. (2017) find systematically higher masses for low mass galaxies than Deason et al. The masses for most of these low mass galaxies were derived using PNe, which Alabi et al. (2016) showed may underestimate the true galaxy mass compared to other tracers of mass (i.e. GCs and X-rays). Here we use the Alabi et al. (2017) masses which are derived only from GCs. Assuming an NFW-like halo, Alabi et al. (2017) also estimated the total virial mass for each galaxy by extrapolating from the $5 \mathrm{R}_{e}$ mass.

The diffuse hot gas X-ray luminosities in the $0.3-8 \mathrm{keV}$ band are taken from the work of KF15. They have carefully removed the contribution from discrete sources such as low mass X-ray binaries (Fabbiano 2006) to the total X-ray luminosity, leaving the diffuse gas contribution $\mathrm{L}_{X, \text { Gas }}$. A correction to bolometric would increase the X-ray luminosities by 0.08 dex on average. Most of the $\mathrm{X}$-ray data come from Chandra observations. However, for some high mass galaxies the X-ray emission is particularly extended (e.g. NGC 4374, 4486, 4649, 5846) and in those cases ROSAT data from O'Sullivan, Forbes \& Ponman (2001), corrected to the Chandra energy band, are used. Although the contribution from discrete sources in the ROSAT data can not be subtracted as accurately as it can for Chandra data, their contribution is only about $1 \%$ of the diffuse gas luminosity for these high mass galaxies (see O'Sullivan, Forbes \& Ponman 2001). For further details see KF15. Here we make a very small correction to the $\mathrm{KF} 15 \mathrm{~L}_{X, \text { Gas }}$ luminosities for the distances used in the SLUGGS survey (Brodie et al. 2014). The KF15 compilation did not include several galaxies that appear in the Alabi et al. (2017) study. Here we also include the X-ray luminosities for NGC 720, NGC 1316 and NGC 3115 from Boroson et al. (2011), and for NGC 5128 from KF13. Su et al. (2014) conducted a detailed XMM and Chandra study of NGC 1400. As well as the X-ray emission centred on NGC 1400, they detected an enhanced region of X-rays to the NE of the galaxy that they asso-

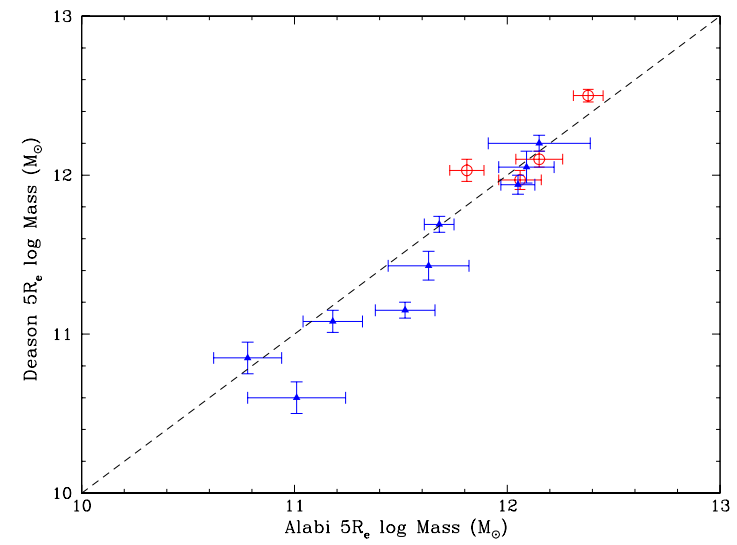

Figure 1. Comparison of total dynamical mass within $5 \mathrm{R}_{e}$ from Deason et al. (2012) with that from Alabi et al. (2017) for galaxies used in this work. Deason et al. masses based on globular clusters (GCs) are shown by open red circles, while those using planetary nebulae (PNe) or a combination of $\mathrm{PNe}$ and GCs are shown by filled blue triangles. The dashed line shows the 1:1 relation. The Alabi et al. masses tend to be systematically higher than the Deason et al. masses for lower mass galaxies.

ciated with stripped gas. Here we use the X-ray luminosity centred on NGC 1400 with a small adjustment to our X-ray band and distance, and assume an uncertainty of $20 \%$. We note that the X-ray luminosity would double if the enhanced region were also included. Two galaxies in Alabi et al. (2017) but not included here are NGC 2974 (not observed by Chandra) and NGC 4474 (the Chandra observation was only $5 \mathrm{ksec}$ ).

In Table 1 we list the properties of our sample galaxies. These include the distance-adjusted $\mathrm{L}_{X, G a s}$, the presence of a core or a cusp in the central optical light profile, the local environment of the galaxy, the stellar mass calculated from the $K$-band luminosity after the correction described by Scott et al. (2013) and assuming $\mathrm{M} / \mathrm{L}_{K}=1$, the dynamical mass within $5 \mathrm{R}_{e}$ and an estimate of the total virial mass.

\section{RESULTS AND DISCUSSION}

In Fig. 2 we show the X-ray luminosity against stellar mass, dynamical mass within $5 \mathrm{R}_{e}$ (i.e. on a scale that is comparable to the X-ray extraction radii used by KF15 and one in which our sample galaxies are dominated by dark matter) and virial mass. These measurements and their uncertainties are given in Table 1.

We also show in Fig. 2 model predictions from the cosmological simulations of Crain et al. (2010) and Choi et al. (2015). Crain et al. (2010) used the GIMIC hydro simulations to predict the X-ray gas properties of 458 isolated late-type galaxy halos. A key finding of their work is that the gas is in quasi-hydrostatic equilibrium and that the large scatter in the optical and IR luminosity- $\mathrm{L}_{X}$ relation is largely due to the variation of stellar mass at a given halo mass. In contrast, the halo virial mass- $\mathrm{L}_{X}$ relation is much tighter, since it directly traces the depth of the potential well. We note that the observations are in the 0.3-8 keV energy range, while the Crain et al. simulations predict the $0.5-2 \mathrm{keV}$ range. We expect a small $(<0.1$ $\mathrm{dex}$ ) increase in their predicted X-ray luminosities to account for the wider band in the observations. Crain et al. did not include AGN in their simulations.

AGN, and the X-ray cavities that they form, are now known 
Table 1. Galaxy sample and properties

\begin{tabular}{|c|c|c|c|c|c|c|c|c|}
\hline $\begin{array}{l}\text { Galaxy } \\
\text { [NGC] } \\
\text { (1) }\end{array}$ & $\begin{array}{l}\text { Dist. } \\
{[\mathrm{Mpc}]} \\
(2)\end{array}$ & $\begin{array}{l}\mathrm{R}_{e} \\
{[”]} \\
(3)\end{array}$ & $\begin{array}{l}\text { Core } \\
\text { (4) }\end{array}$ & $\begin{array}{l}\text { Env. } \\
\text { (5) }\end{array}$ & $\begin{array}{l}\log \mathrm{L}_{X, \text { Gas }} \\
{[\mathrm{erg} / \mathrm{s}]} \\
\text { (6) }\end{array}$ & $\begin{array}{l}\log \mathrm{M}_{*} \\
{\left[\mathrm{M}_{\odot}\right]} \\
(7)\end{array}$ & $\begin{array}{c}\log \mathrm{M}\left(<5 \mathrm{R}_{e}\right) \\
{\left[\mathrm{M}_{\odot}\right]} \\
(8)\end{array}$ & $\begin{array}{c}\log \mathrm{M}_{\text {virial }} \\
{\left[\mathrm{M}_{\odot}\right]} \\
(9)\end{array}$ \\
\hline 720 & 26.9 & 35 & 1 & $\mathrm{~F}$ & $40.68^{\dagger}$ & 11.35 & $11.56(0.20)$ & $12.97(0.44)$ \\
\hline 821 & 23.4 & 40 & 3 & $\mathrm{~F}$ & 38.40 & 10.97 & $11.63(0.19)$ & $12.95(0.30)$ \\
\hline 1023 & 11.1 & 48 & 3 & G & 38.79 & 10.98 & $11.21(0.14)$ & $12.54(0.28)$ \\
\hline 1316 & 20.8 & 69 & 2 & $\mathrm{C}$ & $40.70^{\dagger}$ & 11.81 & $12.06(0.14)$ & $13.72(0.44)$ \\
\hline 1399 & 20.9 & 56 & 1 & $\mathrm{C}^{d}$ & 41.73 & 11.51 & $12.15(0.11)$ & $13.62(0.42)$ \\
\hline 1400 & 26.8 & 28 & 1 & G & $39.67^{\S}$ & 11.12 & $11.37(0.23)$ & $12.71(0.35)$ \\
\hline 1407 & 26.8 & 63 & 1 & $\mathrm{G}^{d}$ & 41.14 & 11.60 & $12.06(0.10)$ & $13.58(0.44)$ \\
\hline 2768 & 21.8 & 63 & 2 & G & 39.88 & 11.28 & $11.85(0.13)$ & $13.23(0.39)$ \\
\hline 3115 & 9.4 & 35 & 3 & $\mathrm{~F}$ & $38.38^{\dagger}$ & 10.97 & $11.31(0.14)$ & $12.63(0.28)$ \\
\hline 3377 & 10.9 & 36 & 3 & G & 38.00 & 10.44 & $10.78(0.16)$ & $12.13(0.21)$ \\
\hline 3607 & 22.2 & 39 & 1 & G & 40.20 & 11.29 & $11.40(0.27)$ & $12.78(0.45)$ \\
\hline 3608 & 22.3 & 30 & 1 & G & 39.62 & 10.82 & $11.53(0.33)$ & $12.84(0.38)$ \\
\hline 4278 & 15.6 & 32 & 1 & G & 39.39 & 10.88 & $11.45(0.13)$ & $12.76(0.23)$ \\
\hline 4365 & 23.1 & 53 & 1 & $\mathrm{G}^{d}$ & 39.66 & 11.48 & $12.08(0.10)$ & $13.54(0.42)$ \\
\hline 4374 & 18.5 & 53 & 1 & $\mathrm{C}$ & 40.82 & 11.46 & $12.15(0.24)$ & $13.60(0.46)$ \\
\hline 4459 & 16.0 & 36 & 3 & $\mathrm{C}$ & 39.39 & 10.93 & $11.27(0.33)$ & $12.59(0.41)$ \\
\hline 4472 & 16.7 & 95 & 1 & $\mathrm{C}$ & 41.36 & 11.74 & $12.37(0.10)$ & $13.95(0.46)$ \\
\hline 4473 & 15.2 & 27 & 1 & $\mathrm{C}$ & 39.09 & 10.87 & $11.16(0.19)$ & $12.47(0.27)$ \\
\hline 4486 & 16.7 & 81 & 1 & $\mathrm{C}^{d}$ & 42.93 & 11.53 & $12.38(0.07)$ & $13.87(0.43)$ \\
\hline 4494 & 16.6 & 49 & 3 & G & 39.11 & 11.02 & $11.18(0.14)$ & $12.51(0.27)$ \\
\hline 4526 & 16.4 & 45 & - & $\mathrm{C}$ & 39.45 & 11.23 & $11.54(0.18)$ & $12.90(0.39)$ \\
\hline 4564 & 15.9 & 20 & 3 & $\mathrm{C}$ & 38.59 & 10.58 & $11.01(0.23)$ & $12.35(0.27)$ \\
\hline 4636 & 14.3 & 89 & 1 & $\mathrm{G}^{d}$ & 41.50 & 11.13 & $11.81(0.08)$ & $13.16(0.33)$ \\
\hline 4649 & 16.5 & 66 & 1 & $\mathrm{C}$ & 41.22 & 11.55 & $12.05(0.08)$ & $13.55(0.43)$ \\
\hline 4697 & 12.5 & 62 & 3 & G & 39.36 & 11.03 & $11.52(0.14)$ & $12.85(0.27)$ \\
\hline 5128 & 3.8 & 305 & - & $\mathrm{G}^{d}$ & $40.20^{*}$ & 10.94 & $11.68(0.07)$ & $13.02(0.23)$ \\
\hline 5846 & 24.2 & 59 & 1 & $\mathrm{G}^{d}$ & 41.70 & 11.40 & $12.09(0.13)$ & $13.52(0.39)$ \\
\hline 5866 & 14.9 & 36 & - & G & 39.40 & 10.97 & $11.10(0.40)$ & $12.42(0.47)$ \\
\hline 7457 & 12.9 & 36 & 3 & $\mathrm{~F}$ & 38.08 & 10.28 & $11.05(0.21)$ & $12.42(0.24)$ \\
\hline
\end{tabular}

Notes: columns are (1) galaxy name, (2) distance, (3) effective radius, (4) 1=core, $2=$ intermediate, $3=$ cusp central light profile from Krajnovic et al. (2013) and Lauer et al. (2007), (5) Environment: $\mathrm{F}=$ field, $\mathrm{G}=$ group, $\mathrm{C}=$ cluster, ${ }^{d}=$ central dominant galaxy, (6) $\log \mathrm{X}$-ray gas luminosity in the $0.3-8 \mathrm{keV}$ band from KF15 (except $\dagger=$ Boroson et al. (2011), $*=\mathrm{KF} 13, \S=$ Su et al. (2014), (7) log total stellar mass from the $K$-band luminosity (an uncertainty of \pm 0.2 dex allows for reasonable variations in IMF, age and metallicity), (8) log dynamical mass within $5 \mathrm{R}_{e}$ and uncertainty, (9) Estimated total virial mass and uncertainty (data for columns 7-9 come from Alabi et al. 2017).

to have an important effect on the hot gas halos around early-type galaxies (McNamara \& Nulsen 2007; Diehl et al. 2008). If an AGN imparts sufficient mechanical energy to the halo gas it can result in an outflow which reduces the overall gas density $\left(n_{e}\right)$ leading to a significant reduction in the X-ray luminosity (as $\mathrm{L}_{X, \text { Gas }} \propto n_{e}^{2}$ ). The Choi et al. (2015) simulations incorporate AGN mechanical heating from a wind for a subsample of 20 ETGs from the cosmological zoom simulations of Oser et al. (2010). Compared to their simulations without AGN, and AGN with pure thermal heating, AGN with mechanical heating produce $X$-ray luminosities that are $100-1000 \times$ lower for a given galaxy. The simulated galaxies are well matched in stellar mass to that of the SLUGGS sample, and they predict $\mathrm{L}_{X, \text { Gas }}$ in the same X-ray energy band as the observed data.

In both the Choi et al. and Crain et al. cosmological simulations the hot gas is initially heated to the virial temperature by shocks as it collapses in the forming dark matter halos. In the case of the Choi et al. simulation, feedback from AGN provides additional heating (which reduces the overall X-ray luminosity since the gas becomes more diffuse due to an outflow).

The left panel of Fig. 2 shows the total stellar mass vs X-ray luminosity (we note that using the stellar mass within $5 \mathrm{R}_{e}$ would give similar results as it is $\sim 90 \%$, or $0.05 \mathrm{dex}$, of the total stel- lar mass for a typical galaxy). The model predictions of Crain et al. (2010) for late-type galaxies show a large range in X-ray luminosity for a given stellar mass. The observations for our ETGs reveal a factor of 100-1000 $\times$ range in $\mathrm{L}_{X, \text { Gas }}$ at a given stellar mass and are generally located on the right hand edge of the Crain et al. distribution. On the other hand, the observations are well matched by the predictions from Choi et al. (2015), albeit over the limited mass range of their simulations. We suggest that Crain et al. overpredicted the X-ray luminosities appropriate for ETGs at a given stellar mass due to the absence of AGN in their model. We return to this point below.

In the middle panel we show the dynamical mass within 5 $\mathrm{R}_{e}$ vs X-ray luminosity. Over this radial range, the galaxies are dark matter dominated (Alabi et al. 2016). A similar trend was seen by KF13 from the X-ray emission of 14 ETGs. To determine the best fit we have applied a bisector linear regression with errors determined from bootstrap resampling. This fit is shown in the middle panel of Fig. 2. This fit is consistent with that found by KF13 (who used masses from Deason et al. 2012), but shifted to slightly higher masses for low mass galaxies as indicated in Fig. 1. The best fit has a scatter of 0.5 dex about the relation: 


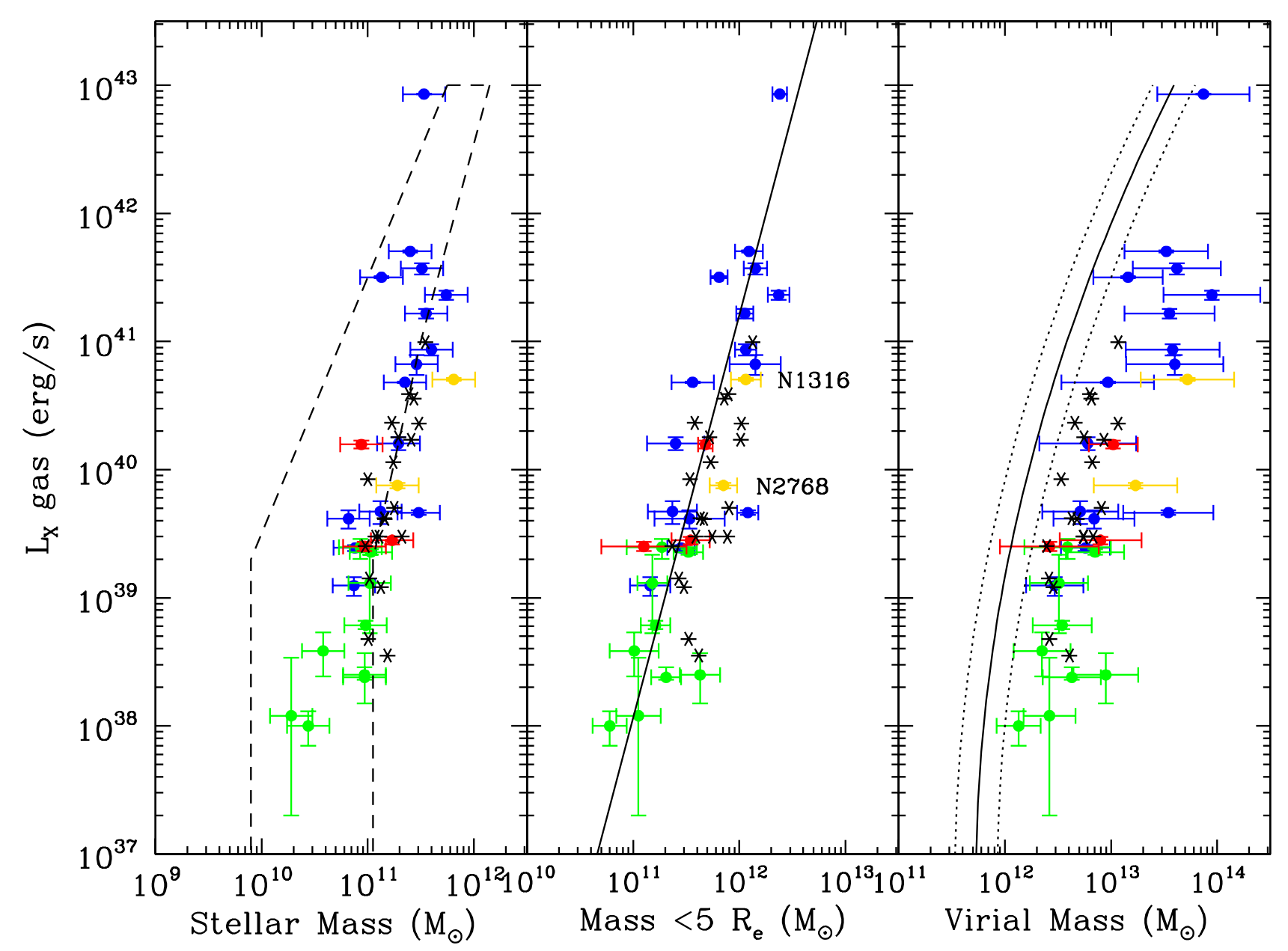

Figure 2. Diffuse gas X-ray luminosity $(0.3-8 \mathrm{keV})$ vs galaxy mass. Early-type galaxies are colour-coded by their central optical light profile $($ blue $=$ core, gold $=$ intermediate, green $=$ cusp, red $=$ unknown). Model galaxies from Choi et al. (2015) are shown as stars. Left panel shows the total stellar mass, derived from the K-band luminosity, with an assumed 0.2 dex uncertainty. The dashed lines show the approximate boundary for the galaxy models of Crain et al. (2010) in the 0.5-2 keV X-ray band. Middle panel shows the dynamical mass within a fixed radius of $5 \mathrm{R}_{e}$. The intermediate profile galaxies NGC 2768 and NGC 1316 are highlighted. The solid line shows a weighted linear fit to the observations. Right panel shows the estimated total virial mass. The solid and dotted curves show the approximate relation and scatter for the galaxy models of Crain et al. (2010) in the 0.5-2 keV X-ray band. Both core and cuspy galaxies follow a similar trend of X-ray luminosity with galaxy mass. [This figure is best viewed in colour.]

$\log \mathrm{L}_{X, \text { Gas }}=3.13( \pm 0.32) \log$ Mass $\left(<5 \mathrm{R}_{e}\right)+3.64( \pm 3.67)$

We also show the simulated galaxies of Choi et al. (2015) in the middle panel, and although they only cover a limited range they are well matched to the observations (Crain et al. did not predict the mass within $5 \mathrm{R}_{e}$ ). The Choi et al. simulations support the interpretation that the data are well represented by a single linear relation. We note that Choi et al. compared their predictions with the best fit relation of KF13 (which used masses from Deason et al. 2013) in their figure 4 and showed that their $\mathrm{L}_{X, G a s}$ values are systematically too low for a given mass. (When using velocity dispersion as a mass proxy the agreement was very good. With our larger data set and the use of (systematically higher) masses from Alabi et al. (2017) their predictions now agree very well with the observations of $\mathrm{L}_{X, \text { Gas }}$ and mass within $5 \mathrm{R}_{e}$.

In the right panel of Fig. 2 we show the total virial mass vs
X-ray luminosity. The virial mass of the observed ETGs has been estimated from the dynamical mass measured at $5 \mathrm{R}_{e}$ and extrapolated to the virial radius assuming a Navarro, Frenk \& White (1997) dark matter profile (see Alabi et al. 2017 for details). Because the ratio of virial mass to dynamical mass within $5 \mathrm{R}_{e}$ varies (due to a non-linear stellar mass to halo mass relation), we expect $\mathrm{L}_{X, \text { Gas }}$ to reveal a non-linear relation with virial mass. A non-linear relation can be seen in the observations shown in the right panel of Fig. 2 .

We also show the 20 model galaxies from Choi et al. (2015) and the Crain et al. (2010) predicted trend, and approximate scatter, for their 458 model galaxies. Crain et al. showed that the scatter in the X-ray luminosity relation using the virial mass compared to the stellar mass is much reduced, and argued that the virial mass is the primary driver of the X-ray luminosity from galaxy halos. Compared to our sample of ETGs, the Crain et al. model predic- 
tions appear to provide an upper envelope; their $\mathrm{L}_{X, \text { Gas }}$ values need to be reduced by at least an order of magnitude to match the observations of ETGs. Choi et al. compared their predicted $\mathrm{L}_{X, \text { Gas }}$ values with virial masses for galaxies and groups from Mathews et al. (2006). Once again, the Choi et al. (2015) simulations predicted $\mathrm{L}_{X, \text { Gas }}$ values that are too low at a given mass (their figure 4). However, with our updated measurements their predictions now reveal a good match to the observations of $\mathrm{L}_{X, \text { Gas }}$ and virial mass.

We note that several of the galaxies in our sample show "hot cores' (i.e. central gas temperatures that are hotter than the surrounding gas) when spatially resolved temperture profiles are available, e.g. NGC 1316 (Kim \& Fabbiano 2013), NGC 3115 (Wong et al. 2011), NGC 4278 (Pellegrini et al. 2012) and NGC 4649 (Paggi et al. 2014). These hot cores are thought to be due to recent heating by the AGN and/or associated radio jets (e.g. Paggi et al. 2014). The influence of AGN may extend down to our lowest stellar masses of $10^{10} \mathrm{M}_{\odot}$ (Cheung et al. 2016).

$\mathrm{KF} 13$ found that the most X-ray luminous ( $\left.\log \mathrm{L}_{X, G a s}>40\right)$ galaxies (i.e. 7 galaxies after excluding NGC 4374 which deviated significantly from their trend) revealed significantly less scatter than the 6 low X-ray luminosity galaxies. With our sample of 29 galaxies, and a new $5 \mathrm{R}_{e}$ dynamical mass for NGC 4374, we find it lies well within the distribution of the $\mathrm{X}$-ray luminous galaxies. We note that the $\sim 3$ Gyr old merger remnant galaxy NGC 1316 (Fornax A) also lies within the scatter of the general trend.

Using a sample of 61 galaxies, KF15 were able to explore the $\mathrm{L}_{X, \text { Gas }}-\mathrm{T}_{X}$ relation subdivided by the shape of their central optical profile, i.e. core or cusp like. They found that core galaxies, which tend to be X-ray luminous, have a very tight relation. In contrast, low X-ray luminosity cusp galaxies revealed no clear correlation between $\mathrm{L}_{X, G a s}$ and $\mathrm{T}_{X}$. In Fig. 2 we have colour-coded our sample by their central optical profile (see Table 1). The galaxies are classified as core, cusp, intermediate or unknown surface brightness profile from Krajnovic et al. (2013) or Lauer et al. (2007). We note that there is some disagreement in the literature about the central profile in some galaxies, e.g. Dullo \& Graham (2013) suggested that NGC 4473 does not contain a central core but rather a disk.

In all three panels, the sample divides clearly between core (high X-ray luminosity) and cusp (low X-ray luminosity) galaxies, with 5 additional galaxies ( 3 unknown and 2 intermediate profiles). The sample divides more clearly in terms of $\mathrm{L}_{X, \text { Gas }}$ than mass (see also Pellegrini 2005). We note that the central profile shape correlates very strongly with central rotation, i.e. core galaxies $\approx$ slow rotators, and cusp galaxies $\approx$ fast rotators (Krajnovic et al. 2013).

Focusing on the middle panel, the 17 core galaxies reveal a somewhat tighter relation than the 9 cuspy galaxies. We find a pvalue for the Spearman rank correlation test of 0.0007 for the core galaxies, whereas the cusp galaxies have a value of 0.26 indicating almost no relation. A bisector fit to only the cuspy galaxies also suggests no statistically significant relation. This supports the findings of KF15 and suggests that SN heating may play an increased role in cuspy (low mass) ETGs (David et al. 2006). So although the X-ray luminosity of both core (high X-ray luminosity) and cuspy (low X-ray luminosity) ETGs are primarily determined by total mass, and hence the depth of the potential well, other factors may play a role for the lower mass galaxies.

\section{CONCLUSIONS}

Using new dynamical masses we find a strong linear relationship between the diffuse gas X-ray luminosity for a sample of 29 nearby massive early-type galaxies and their total dynamical mass within $5 \mathrm{R}_{e}$. This result supports the earlier analysis by KF13 based on 14 galaxies. We also show that the cosmological simulations of Choi et al. (2015), which incorporate AGN mechanical heating, now agree with observations of the X-ray luminosity and mass within $5 \mathrm{R}_{e}$. This good agreement with model galaxies in a cosmological framework supports the idea that the diffuse X-ray luminosity is primarily driven by the depth of a galaxy's potential well, with a significant contribution from AGN mechanical heating. Supernova heating may contribute in lower mass galaxies.

\section{ACKNOWLEDGEMENTS}

We thank R. Crain for useful discussion and E. Choi for her model data. We also thank the referee for useful feedback (of the non-AGN kind). DAF thanks the ARC for financial support via DP130100388. This work was supported by NSF grant AST1211995 and NASA contract NAS8-03060 (CXC). GF thanks the Aspen Center for Physics, funded by NSF grant 1066293, for their hospitality while this paper was completed. DWK acknowledges support by the Chandra GO grant AR4-15005X and by 2014 Smithsonian Competitive Grant Program for Science.

\section{REFERENCES}

Alabi A., et al. 2016, MNRAS, in press Alabi A., et al. 2017, in prep

Boroson B., Kim D.-W., Fabbiano G., 2011, ApJ, 729, 12

Brodie J. P., et al., 2014, ApJ, 796, 52

Cheung E., et al., 2016, Natur, 533, 504

Choi E., et al. 2015, MNRAS, 449, 4105

Crain R. A., et al. 2010, MNRAS, 407, 1403

Diehl S., Li H., Fryer C. L., Rafferty D., 2008, ApJ, 687, 173-192

Dullo B. T., Graham A. W., 2013, ApJ, 768, 36

David L. P., et al. 2006, ApJ, 653, 207

Deason A. J., et al. 2012, ApJ, 748, 2

Fabbiano G., 2006, ARA\&A, 44, 323

Goulding A. D., et al., 2016, arXiv:1604.01764

Kim, D.-W., Fabbiano, G. 2003, ApJ, 586, 826

Kim D.-W., Fabbiano G., 2013, ApJ, 776, 116 (KF13)

Kim D.-W., Fabbiano G., 2015, ApJ, 812, 127 (KF15)

Krajnović D., et al., 2013, MNRAS, 433, 2812

Lauer T. R., et al., 2007, ApJ, 664, 226

Mathews W. G., et al. 2006, ApJ, 652, L17

McNamara B. R., Nulsen P. E. J., 2007, ARA\&A, 45, 117

Navarro J. F., Frenk C. S., White S. D. M., 1997, ApJ, 490, 493

Oser L., et al. 2010, ApJ, 725, 2312

O’Sullivan E., Forbes D. A., Ponman T. J., 2001, MNRAS, 328, 461

Paggi A., et al. 2014, ApJ, 787, 134

Pellegrini S., 2005, ApJ, 624, 155

Pellegrini S., et al. 2012, ApJ, 758, 94

Pota V., et al., 2013, MNRAS, 428, 389

Sarzi M., et al., 2013, MNRAS, 432, 1845

Scott N., Graham A. W., Schombert J., 2013, ApJ, 768, 76

Su Y., Gu L., White R. E., III, Irwin J., 2014, ApJ, 786, 152

Sun M., Donahue M., Voit G. M., 2007, ApJ, 671, 190

Watkins L. L., Evans N. W., An J. H., 2010, MNRAS, 406, 264

White S. D. M., Frenk C. S., 1991, ApJ, 379, 52

Wong K.-W., et al. 2011, ApJ, 736, L23 